\title{
ORIGINAL
}

\section{ESTIMACIÓN DE LA CARGA DE ENFERMEDAD POR INCAPACIDAD LABORAL PERMANENTE EN ESPAÑA DURANTE EL PERÍODO 2009-2012 (*)}

\author{
María Andrée López (1,2,3), Xavier Duran (1,2,3), Jordi Alonso (1,3), José Miguel Martínez (1, 2,3), \\ Mireia Espallargues $(1,4)$ y Fernando G. Benavides $(1,2,3)$.
}

(1) Consorcio de Investigación Biomédica en Red, Epidemiología y Salud Pública (CIBERESP). Evaluación de Servicios de Salud.

(2) Centro de Investigación en Salud Laboral. Universitat Pompeu Fabra. Barcelona.

(3) IMIM-Institut Hospital del Mar d'Investigacions Mèdiques. Barcelona.

(4) Agència de Qualitat i Avaluació Sanitàries (AQuAS).

(*) Este trabajo recibió ayuda económica de Consorcio de Investigación Biomédica en Red,Epidemiología y Salud Pública (CIBERESP).Evaluación de Servicios de Salud (Programa 6).

Los autores declaran no tener conflictos de intereses.

\section{RESUMEN}

Fundamentos: La incapacidad laboral permanente (IP) supone una importante carga social y económica. El objetivo de este estudio fue estimar la carga de enfermedad debida a la IP en España.

Métodos: A partir de la Muestra Continua de Vidas Laborales (MCVL) se imputó como diagnóstico de la IP (2009-2012) el de la incapacidad temporal (IT) iniciada en 2009 en los individuos que cumplieron dos criterios: larga duración de la IT y corta duración del período entre la IT y la IP. Para los casos con diagnóstico imputado se calculó la carga de enfermedad por IP a partir de los años potenciales de vida laboral perdidos (APVLP), definidos como la diferencia entre la edad de jubilación (65 años) y la edad de inicio de la IP.

Resultados: Según la MCVL, $163.135(13,6 \%)$ personas sufrieron de IT en 2009 , de las cuáles a $4.738(0,39 \%)$ se le reconoció una IP entre 2009-2012. Se pudo establecer el diagnóstico de IP en 3.073 (64,9\%) casos. Las causas más frecuentes de IP fueron los trastornos musculo-esqueléticos $(27,4 \%)$ y mentales $(11,6 \%)$ que, asimismo, produjeron mayor cantidad de APVLP por IP: $11.086(26,5 \%)$ y $7.052(16,9 \%)$, respectivamente. Los trastornos mentales destacaron por producir IP a edades más jóvenes (mediana $=49$ años).

Conclusiones: Los trastornos mentales y los musculo-esqueléticos representaron la mayor carga de enfermedad en individuos en situación de IP.

Palabras clave: Costo de enfermedad. Seguro por discapacidad. Seguridad Social. Ausencia por enfermedad. Sistema de registros.

\section{Correspondencia}

María Andrée López

Centro de Investigación en Salud Laboral

Universitat Pompeu Fabra

Barcelona

Correo electrónico: andree.gomez01@estudiant.upf.edu

\section{ABSTRACT \\ Estimating the Burden of Disease Due to Permanent Disability in Spain during the Period 2009-2012}

Background: Permanent disability (PD) is a major social and economic burden. The aim of this study was to determine the burden of disease due to PD

Methods: Using data from the Continuous Working Life Sample (CWLS) -a random sample consisting of $4 \%$ of the active members affiliated to the Social Security (SS) System in Spain (1.1 million members) which data come from administrative registries from the SS- the diagnosis of PD (2009-2012) was established from the information on the temporary sickness absence (TSA) episodes from 2009. The diagnoses were imputed if two criteria were fulfilled: long duration of TSA and short period of time between the TSA episode and PD. The burden of disease was estimated for cases with an established diagnosis of PD by using the potential years of working life lost (PYWLL), estimated as the difference between the legal retirement age (65 years) and the age at the start of a PD.

Results: In the CWLS, 163,135 (13.6\%9 individuals suffered a TSA episode in 2009 , out of which 4,738 were granted a PD pension in the period of 2009-2012. The diagnosis was established for 3,073 $(64.9 \%)$ cases. A large percentage of PD was produced by musculoskeletal $(27.4 \%)$ and mental disorders $(11.6 \%)$, likewise these diagnoses produced more PYWLL due to a PD: $11,086(26.5 \%)$ and 7,052 (16.9\%), respectively. Mental disorders produced PD at younger ages (median= 49 years of age).

Conclusion: Mental and musculoskeletal disorders represent a major burden of disease produced by PD and measured by PYWLL. The diagnoses of PD should become available in order to estimate with more accuracy the PYWLL produced by PD and specific diagnoses.

Keyword: Cost of illness. Insurance disability. Social security. Sick leave. Registries. 


\section{INTRODUCCIÓN}

El incremento de la esperanza de vida, fruto de las mejoras en las condiciones de vida y de trabajo junto a las de la asistencia sanitaria, tiene como una de sus consecuencias el progresivo envejecimiento de la población y, con ello, el incremento de la prevalencia de enfermedades crónicas: cardiovasculares, cáncer, mentales y musculoesqueléticas ${ }^{1}$. La cronicidad de estas enfermedades se manifiesta precozmente durante la edad activa y más en la medida que esta se va alargando, consecuencia de las reformas de las pensiones ${ }^{2}$, lo que provoca una mayor carga para la sociedad, tanto en términos de asistencia como de protección social ${ }^{3}$.

La situación de incapacidad permanente (IP) reconocida por el Instituto Nacional de la Seguridad Social (INSS) cuando un problema de salud afecta a una persona afiliada que cumple determinados requisitos legales, se relaciona de manera principal con las enfermedades crónicas. La IP puede ser de origen común o profesional y se reconoce por parte de un Equipo de Valoración de Incapacidad (en cada provincia) cuando el problema de salud que sufre incapacita al trabajador/a de manera previsiblemente definitiva para su trabajo (grados de IP "total" o "absoluta") e incluso para realizar otras tareas de la vida diaria ("gran invalidez") $)^{4,5}$, dando lugar a una prestación económica cuya cuantía depende del grado de IP reconocido. Como es previsible, la mayoría de las IP comienzan con al menos un episodio de incapacidad temporal (IT), justificada por un médico que certifica el diagnóstico $^{4}$ y que legalmente puede durar hasta un año, prorrogable por seis meses siempre que se prevea la recuperación durante ese tiempo, y en caso contrario la IT sería evaluada con el fin de reconocer o no la situación de IP o producir el alta ${ }^{6}$.

En España, la incidencia de IP de origen común durante 2004-2007 fue de 36,3 casos por 10.000 trabajadores/año y aumenta en personas mayores de 55 años con una incidencia de 133,6 casos por 10.000 trabajadores/año ${ }^{7}$. En 2012 se registraron 943.296 casos de personas que recibían prestaciones económicas por IP, lo que supuso un gasto anual para la Seguridad Social de 11.000 millones de euros ${ }^{8}$. Estos datos muestran que la IP es un importante problema de salud laboral y salud pública ${ }^{3,9,10}$ que, sin embargo, está poco estudiado en España, entre otras razones por la falta del diagnóstico médico en los registros administrativos disponibles para los investigadores.

La medición de la carga de la enfermedad por IP a través del cálculo de los años potenciales de vida laboral perdidos (APVLP), como diferencia entre la edad de inicio de la IP y la edad de jubilación actual (65 años), para cada enfermedad o grupo de enfermedades ofrece diversas ventajas. Entre ellas que es fácil de calcular, además de rápidamente comprensible, permitiendo comparar entre las enfermedades que producen la IP, una vez se ha fijado el límite superior (edad de jubilación). Asimismo, los APVLP proporcionan una medida del coste social de la IP en forma de subsidios que aporta la seguridad social y de pérdida en la productividad en las empresas por la descapitalización de los recursos humanos que esta retirada prematura del mercado laboral representa. En la actualidad supone un grave problema para la mayoría de los países europeos ${ }^{3}$, incluida España $^{7}$, dado el paulatino envejecimiento de la población laboral.

El objetivo de este estudio fue estimar en España la carga de enfermedad producida por la IP en una muestra de sujetos en situación de alta o pensionistas de la Seguridad Social.

\section{MATERIAL Y MÉTODOS}

Este estudio se basó en la Muestra Continua de Vidas Laborales (MCVL) de 20092012, en la que se registra el inicio de las IP pero sin diagnóstico médico, y su conexión 
con los registros de IT del año 2009 procedentes del Instituto Nacional de Seguridad Social (INSS), en el que sí se recoge el diagnóstico al alta de cada episodio de IT y su duración. La conexión se realizó por medio de un identificador personal que administra la propia seguridad social, sin que pueda ser identificado el individuo. La conexión permitió el seguimiento hasta el año 2012 de quienes tuvieron un episodio de IT en 2009, para comprobar si se les reconoció una IP durante ese periodo. Se tomó en cuenta a todas las IP, tanto de origen común como profesional.

Desde 2004 la Dirección General de Ordenación de la Seguridad Social extrae anualmente de manera aleatoria una muestra del $4 \%$ de sujetos cotizantes y/o pensionistas $(\sim 1,1$ millón de personas al año) de la seguridad social para elaborar la MCVL y distribuirla para su uso en la investigación. Los datos de la MCVL proceden de varios registros administrativos de la seguridad social, entre ellos el de prestaciones y cotizaciones. Al ser una muestra anual tipo panel en la que se puede seguir a los individuos a lo largo de los años, siempre que continúen como afiliados en alta a la seguridad social, la MCVL permite realizar estudios de cohorte ${ }^{11}$. Para este estudio se identificó una sub-muestra de 163.135 personas de la MCVL de 2009 que registraron al menos un episodio de IT iniciado en 2009 de acuerdo a los registros del INSS $^{12}$. Se realizó un seguimiento hasta 2012 en la MCVL y se identificó a quienes se les concedió una prestación por IP. De todas las personas con una IT, se dispuso de su diagnóstico de alta certificado por el médico en el $95 \%$ de los casos y solo en $8.222(5,0 \%)$ se utilizó el diagnóstico de la baja certificado por el médico, aunque en el estudio se utilizó el del alta por considerarlos más preciso. El diagnóstico se clasificó de acuerdo a la Clasificación Internacional de Enfermedades versión 9 (CIE-9).

Para imputar el diagnóstico de IP iniciada entre 2009 y 2012 se utilizó el de la IT ocu- rrida en 2009 teniendo en cuenta criterios relacionados con la duración del episodio de la IT y del tiempo transcurrido entre la finalización de la IT y el inicio de la IP. Tras varias simulaciones se establecieron diferentes puntos de corte. En relación a la duración de la IT, varios estudios observan un aumento en la probabilidad de desarrollar una IP al incrementar la duración de la IT ${ }^{13-}$ 18. Este tiempo de duración de la IT se agrupó en tres categorías con su respectiva puntuación: menos de 4 meses ( 0 puntos), entre 4 y 6 meses ( 1 punto) y más de 6 meses (2 puntos). El segundo criterio se basó en la presunción que si el tiempo entre la finalización de IT y el inicio de IP es corto, es más probable que la IT esté relacionada con la IP. Así el período entre IT e IP se agrupó en tres categorías: más de 12 meses (0 puntos), entre 4 y 12 meses (1 punto) y menos de 4 meses (2 puntos). En 40.823 (25\%) individuos con más de una IT iniciada en 2009 se utilizó el último episodio de IT y siempre se tomó en cuenta el diagnóstico de la IT de más larga duración para calcular los puntos, pues una IT más larga presupone un problema más grave de salud.

Ambos criterios tomaron en cuenta el proceso de aprobación de la prestación económica por IP, según la cual las personas que sufren una IT prolongada en el tiempo deben asistir a revisiones médicas con el propósito de determinar si se reconoce o no el derecho a ella. Una vez sumadas las puntuaciones de ambos criterios, si se obtenía una puntuación de 2-4 se consideró que el diagnóstico de la IT era probable que fuera el de la IP e improbable si la puntuación era de 0-1.

También se investigó si había habido múltiples episodios de IT anteriores a la IP en el 2009 , pero no se encontró una relación entre este criterio y desarrollar una IP, por lo cual no se incluyó en el análisis.

En los casos en que se pudo utilizar el diagnóstico IT como el de IP (casos de diagnóstico probable) se calcularon los APVLP 
por IP mediante la diferencia entre la edad de inicio de la IP y la edad de jubilación actual (65 años). También se estimó la edad mediana de inicio de IP y sus percentiles 25 y 75 . La suma total de los APVLP para cada grupo de enfermedad permitió identificar los diagnósticos que produjeron una mayor carga de enfermedad por IP.

\section{RESULTADOS}

De acuerdo a los registros del Instituto Nacional de la Seguridad Social (INSS), $163.135(13,6 \%)$ individuos de la MCVL tuvieron al menos un episodio de IT en el 2009. Tras su seguimiento hasta 2012 se identificó a $4.738(2,9 \%)$ individuos a los que se les reconoció una IP (figura 1). $3.525(74,4 \%)$ personas en IP tuvieron solamente un episodio de IT. Al aplicar los criterios estipulados de duración de IT y período entre IT e IP se pudo imputar el diagnóstico de IT (2009) como probable diagnóstico de IP (2009-2012) en 3.073 $(64,9 \%)$ casos. El análisis de APVLP se realizó en estos últimos casos.

En la tabla 1 se puede observar la distribución de IT en 2009 por grupos diagnósticos y de ellas el número de casos que desarrollaron una IP entre 2009 y 2012. De los 2.190 individuos diagnosticados de neoplasia registrados en 2009, finalizaron en IP 266 (12,2\%). Seguidas de las enfermedades cardiovasculares donde a $335(9,6 \%)$ personas en IT se les reconoció la situación de IP. Por el contrario, los trastornos musculo-esqueléticos fueron la causa más frecuente de IT, $30.855(18,9 \%)$ personas de las que $1.296(4,2 \%)$ acabaron en situaciín

Figura 1

Diagrama de inclusión en el estudio de carga de enfermedad por incapacidad permanente en la Muestra Continua de Vidas Laborales (MCVL) con datos de incapacidad temporal (2009) del Instituto de Seguridad Social (INSS)

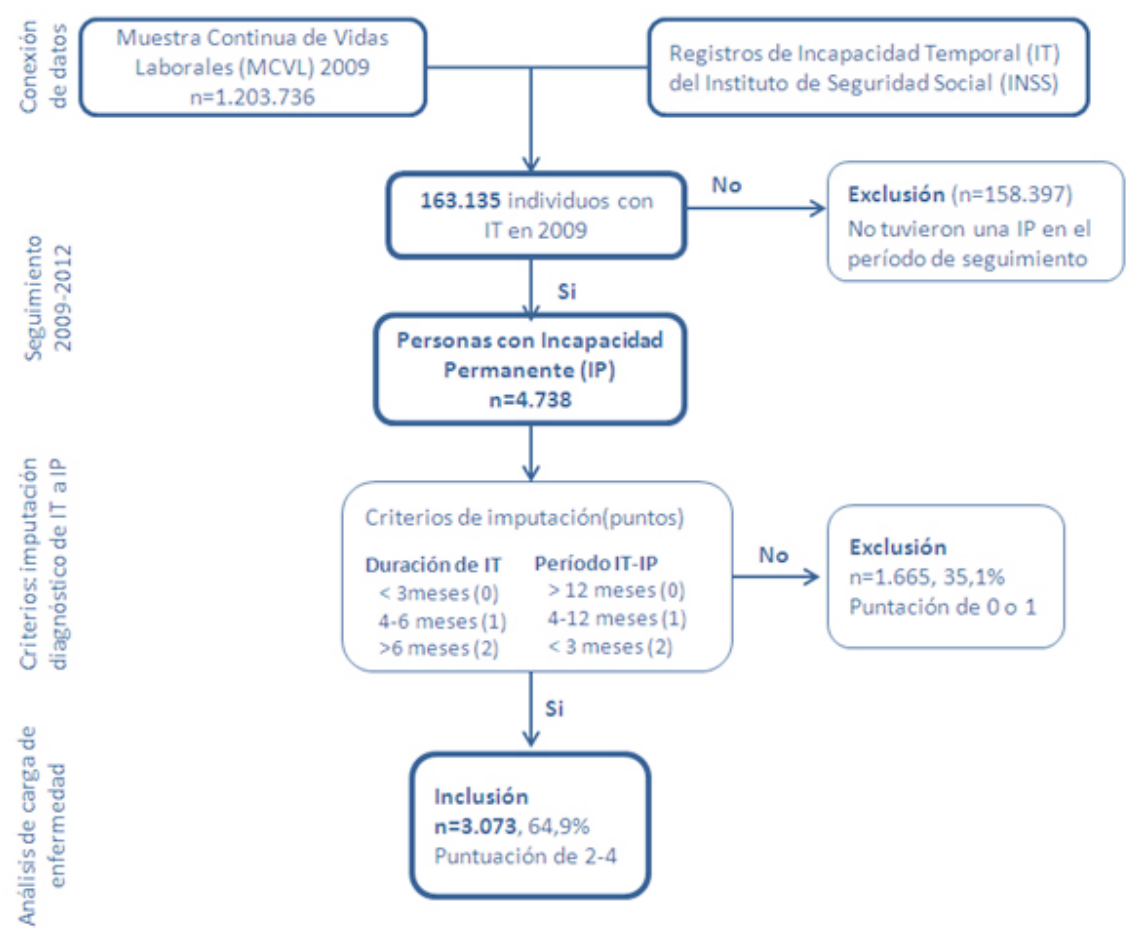




\section{Tabla 1}

Distribución por grupos diagnósticos de los episodios de incapacidad temporal (IT) iniciados en 2009 y que evolucionaron hacia una incapacidad permanente (IP) entre 2009-2012. MCVL 2009-2012

\begin{tabular}{|c|c|c|c|c|}
\hline \multirow{2}{*}{ Diagnósticos (CIE-9) IT } & IT & \multicolumn{3}{|c|}{ IP } \\
\hline & $\mathrm{n}(\%)$ & $\mathrm{n}$ & $\% *$ & $\%^{\dagger}$ \\
\hline Infecciosas y parasitarias (001-139) & $13.807(8,5)$ & 198 & 1,4 & 4,2 \\
\hline Neoplasias (140-239) & $2.190(1,3)$ & 266 & 12,2 & 5,6 \\
\hline Trastornos endocrinos (240-279) & $1.787(1,1)$ & 67 & 3,8 & 1,4 \\
\hline Sangre y órganos hematopoyéticos (280-289) & $266(0,2)$ & 14 & 5,3 & 0,3 \\
\hline Trastornos Mentales (290-319) & $9.958(6,1)$ & 548 & 5,5 & 11,6 \\
\hline Sistema Nervioso y sentidos (320-389) & $5.662(3,5)$ & 235 & 4,2 & 5,0 \\
\hline Cardiovasculares (390-459) & $3.503(2,2)$ & 335 & 9,6 & 7,1 \\
\hline Aparato respiratorio (460-519) & $29.453(18,1)$ & 306 & 1,0 & 6,5 \\
\hline Aparato digestivo (520-579) & $8.264(5,1)$ & 156 & 1,9 & 3,3 \\
\hline Aparato genitourinario (580-629) & $3.129(1,9)$ & 65 & 2,1 & 1,4 \\
\hline Embarazo, parto y puerperio $(630-677)^{\dagger \dagger}$ & $3.445(2,1)$ & 7 & 0,2 & 0,1 \\
\hline Piel y tejido subcutáneo (680-709) & $2.026(1,2)$ & 51 & 2,5 & 1,1 \\
\hline Musculo-esqueléticos (710-739) & $30.885(18,9)$ & 1.296 & 4,2 & 27,4 \\
\hline Anomalías congénitas (740-759) & $204(0,1)$ & 17 & 8,3 & 0,4 \\
\hline Período perinatal (760-779) & $873(0,5)$ & 28 & 3,2 & 0,6 \\
\hline Síntomas, signos y estados mal definidos (780-799) & $14.480(8,9)$ & 373 & 2,6 & 7,9 \\
\hline Lesiones y envenenamientos (800-999) & $19.742(12,1)$ & 423 & 2,1 & 8,9 \\
\hline Intervenciones quirúrgicas (00-99) & $162(0,1)$ & 4 & 2,5 & 0,1 \\
\hline Sin diagnóstico & $13.299(8,2)$ & 349 & 2,6 & 7,4 \\
\hline Total & $163.135(100,0)$ & 4.738 & 2,9 & 100,0 \\
\hline
\end{tabular}

* Porcentaje de IT en 2009 que desarrollaron una IP en 2009-2012, †Porcentaje de IP en 2009-2012, †Complicaciones del embarazo, parto o puerperio

de IP en el período de seguimiento. Si bien son las causas más frecuentes de IP $(27,4 \%)$. Algo similar ocurrió con las lesiones $(8,9 \%)$ y los trastornos mentales $(11,6 \%)$ de las IP.

Al aplicar los criterios definidos se observó que el 54,7\% de todos los casos con IP tuvieron una IT de larga duración, es decir de más de seis meses, y el 37,4\% tuvieron un tiempo entre la IT e inicio de la IP de menos de 4 meses (tabla 2).

Así, se pudo asignar el diagnóstico de IT como probable diagnóstico de la IP a 3.073
$(64,9 \%)$ personas en las que se realizó el análisis de APVLP. El total de APVLP para las personas con IP con diagnóstico imputado fue de 41.834 años (tabla 3). Los trastornos musculo-esqueléticos (11.086 años) $(26,5 \%)$, los trastornos mentales (7.052 años) $(16,9 \%)$ y las lesiones y envenenamientos (4.038 años) (9,7\%) fueron las patologías con mayor número de APVLP. El 50\% de las 402 personas con diagnóstico de trastorno mental iniciaron su IP antes de los 49 años, por lo cual la mediana de los APVLP por IP para este diagnóstico fue de 16 años. 
Tabla 2

Puntuación en cada criterio que determinará la probabilidad que el diagnóstico que produjo la incapacidad temporal (IT) en 2009 sea el diagnóstico de la incapacidad permanente (IP) producida en 2009-2012. N (\%)

\begin{tabular}{|c|c|c|c|c|c|c|c|c|}
\hline \multirow[b]{2}{*}{ Diagnósticos (CIE-9) de incapacidad temporal } & \multicolumn{3}{|c|}{ Duración IT } & \multicolumn{3}{|c|}{ Período entre IT-IP } & \multicolumn{2}{|c|}{ Probabilidad diagnóstico IP* } \\
\hline & $\begin{array}{l}0-3 \text { meses } \\
(0 \text { puntos })\end{array}$ & $\begin{array}{r}4-6 \text { meses } \\
\text { (1 punto) }\end{array}$ & $\begin{array}{l}>6 \text { meses } \\
(2 \text { puntos })\end{array}$ & $\begin{array}{l}>12 \text { meses } \\
(0 \text { puntos })\end{array}$ & $\begin{array}{c}\text { 4-12 meses } \\
\text { (1 punto) }\end{array}$ & $\begin{array}{l}0-3 \text { meses } \\
(2 \text { puntos })\end{array}$ & Improbable & Probable ${ }^{\S}$ \\
\hline Infecciosas y parasitarias (001-139) & $101(51,0)$ & $19(9,6)$ & $78(39,4)$ & $111(56,1)$ & $32(16,2)$ & $55(27,8)$ & $105(6,3)$ & $93(3,0)$ \\
\hline Neoplasias (140-239) & $24(9,0)$ & $37(13,9)$ & $205(77,1)$ & $40(15,0)$ & $59(22,2)$ & $167(62,8)$ & $21(1,3)$ & $245(8,0)$ \\
\hline Trastornos endocrinos (240-279) & $18(26,9)$ & $9(13,4)$ & $40(59,7)$ & $30(44,8)$ & $8(11,9)$ & $29(43,3)$ & $19(1,1)$ & $48(1,6)$ \\
\hline Sangre y órganos hematopoyéticos (280-289) & $3(21,4)$ & $0(0,0)$ & $11(78,6)$ & $3(21,4)$ & $3(21,4)$ & $8(57,1)$ & $2(0,1)$ & $12(0,4)$ \\
\hline Trastornos Mentales (290-319) & $130(23,7)$ & $69(12,6)$ & $349(63,7)$ & $197(36,0)$ & $136(24,8)$ & $215(39,2)$ & $146(8,8)$ & $402(13,1)$ \\
\hline Sistema Nervioso y sentidos (320-389) & $84(35,7)$ & $42(17,9)$ & $109(46,4)$ & $89(37,9)$ & $53(22,6)$ & $93(39,6)$ & $89(5,4)$ & $146(4,8)$ \\
\hline Cardiovasculares (390-459) & $53(15,8)$ & $48(14,3)$ & $234(69,9)$ & $71(21,2)$ & $72(21,5)$ & $192(57,3)$ & $57(3,4)$ & $278(9,1)$ \\
\hline Aparato respiratorio (460-519) & $218(71,2)$ & $25(8,2)$ & $63(20,6)$ & $209(68,3)$ & $37(12,1)$ & $60(19,6)$ & $218(13,1)$ & $88(2,9)$ \\
\hline Aparato digestivo (520-579) & $69(44,2)$ & $17(10,9)$ & $70(44,9)$ & $77(49,4)$ & $25(16,0)$ & $54(34,6)$ & $74(4,4)$ & $82(2,7)$ \\
\hline Aparato genitourinario (580-629) & $18(27,7)$ & $7(10,8)$ & $40(61,5)$ & $25(38,5)$ & $12(18,5)$ & $28(43,1)$ & $20(1,2)$ & $45(1,5)$ \\
\hline Embarazo, parto y puerperio $(630-677)^{\ddagger}$ & $4(57,1)$ & $0(0,0)$ & $3(42,9)$ & $6(85,7)$ & $1(14,3)$ & $0(0,0)$ & $4(0,2)$ & $3(0,1)$ \\
\hline Piel y tejido subcutáneo (680-709) & $26(51,0)$ & $6(11,8)$ & $19(37,3)$ & $29(56,9)$ & $11(21,6)$ & $11(21,6)$ & $25(1,5)$ & $26(0,9)$ \\
\hline Musculo-esqueléticos (710-739) & $365(28,2)$ & $197(15,2)$ & $734(56,6)$ & $527(40,7)$ & $330(25,5)$ & $439(33,9)$ & $422(25,4)$ & $874(28,4)$ \\
\hline Anomalías congénitas (740-759) & $5(29,4)$ & $0(0,0)$ & $12(70,6)$ & $4(23,5)$ & $6(35,3)$ & $7(41,2)$ & $4(0,2)$ & $13(0,4)$ \\
\hline Período perinatal (760-779) & $5(17,9)$ & $5(17,9)$ & $18(64,3)$ & $14(50,0)$ & $7(25,0)$ & $7(25,0)$ & $9(0,5)$ & $19(0,6)$ \\
\hline Síntomas, signos y estados mal definidos (780-799) & $146(39,1)$ & $34(9,1)$ & $193(51,7)$ & $161(43,2)$ & $85(22,8)$ & $127(34,1)$ & $147(8,8)$ & $226(7,4)$ \\
\hline Lesiones y envenenamientos (800-999) & $134(31,7)$ & $61(14,4)$ & $228(53,9)$ & $191(45,2)$ & $90(21,3)$ & $142(33,6)$ & $162(9,7)$ & $261(8,5)$ \\
\hline Intervenciones quirúrgicas (00-99) & $0(0,0)$ & $1(25,0)$ & $3(75,0)$ & $2(50,0)$ & $1(25,0)$ & $1(25,0)$ & $1(0,1)$ & $3(0,1)$ \\
\hline Sin diagnóstico & $137(39,3)$ & $29(8,3)$ & $183(52,4)$ & $155(44,4)$ & $56(16,1)$ & $138(39,5)$ & $140(8,4)$ & $209(6,8)$ \\
\hline Total & $1.540(32,5)$ & $606(12,8)$ & $2.592(54,7)$ & $1.941(41,0)$ & $1.024(21,6)$ & $1.773(37,4)$ & $1.665(100,0)$ & $3.073(100,0)$ \\
\hline
\end{tabular}

*Los porcentajes son de columna y no de fila como los de duración IT y período entre IT-IP, ${ }^{\star}$ Complicaciones del embarazo, parto o puerperio, ${ }^{\dagger}$ Es improbable que el diagnóstico de la IT sea el de la IP por tener una puntuación de 0-1 tomando en cuenta los criterios de imputación, ${ }^{\S}$ Es probable que el diagnóstico de la IT sea el de la IP por tener una puntuación de 2-4 tomando en cuenta los criterios de imputación 
Tabla 3

Años potenciales de vida laboral perdidos (APVLP) debido a una incapacidad permanente (IP) en 2009-2012 en personas con al menos una incapacidad temporal (IT) iniciada en 2009 según diagnóstico

\begin{tabular}{|c|c|c|c|c|c|c|}
\hline \multirow{2}{*}{ Diagnósticos (CIE-9) de la IT } & \multirow{2}{*}{$\begin{array}{l}\text { Edad IP } \\
\text { Mediana }\end{array}$} & \multirow{2}{*}{$\mathrm{n}(\%)$} & \multirow{2}{*}{ APVLP $(\%)$} & \multicolumn{3}{|c|}{ APVLP* } \\
\hline & & & & $\mathrm{P} 25$ & P50 & P75 \\
\hline Infecciosas y parasitarias (001-139) & 55 & $93(3,0)$ & $1.091(2,6)$ & 6 & 10 & 16 \\
\hline Neoplasias (140-239) & 55 & $245(8,0)$ & $2.915(7,0)$ & 5 & 10 & 17 \\
\hline Trastornos endocrinos (240-279) & 54 & $48(1,6)$ & $634(1,5)$ & 6 & 11 & 20 \\
\hline Sangre y órganos hematopoyéticos (280-289) & 56 & $12(0,4)$ & $129(0,3)$ & 2 & 9 & 16 \\
\hline Trastornos Mentales (290-319) & 49 & $402(13,1)$ & $7.052(16,9)$ & 9 & 16 & 25 \\
\hline Sistema Nervioso y sentidos (320-389) & 56 & $146(4,8)$ & $1.825(4,4)$ & 5 & 9 & 19 \\
\hline Cardiovasculares (390-459) & 56 & $278(9,0)$ & $2.950(7,1)$ & 5 & 9 & 15 \\
\hline Aparato respiratorio (460-519) & 55 & $88(2,9)$ & $1.118(2,7)$ & 5 & 10 & 19 \\
\hline Aparato digestivo (520-579) & 51 & $82(2,7)$ & $1.193(2,9)$ & 7 & 14 & 20 \\
\hline Aparato genitourinario (580-629) & 54 & $45(1,5)$ & $608(1,5)$ & 5 & 11 & 23 \\
\hline Embarazo, parto y puerperio $(630-677)^{\dagger}$ & 38 & $3(0,1)$ & $82(0,2)$ & 26 & 27 & 29 \\
\hline Piel y tejido subcutáneo (680-709) & 57 & $26(0,8)$ & $328(0,8)$ & 5 & 8 & 20 \\
\hline Musculo-esqueléticos (710-739) & 54 & $874(28,4)$ & $11.086(26,5)$ & 6 & 11 & 18 \\
\hline Anomalías congénitas (740-759) & 48 & $13(0,4)$ & $260(0,6)$ & 12 & 17 & 28 \\
\hline Período perinatal (760-779) & 54 & $19(0,6)$ & $217(0,5)$ & 4 & 11 & 13 \\
\hline Síntomas, signos y estados mal definidos (780-799) & 54 & $226(7,4)$ & $3.119(7,5)$ & 7 & 11 & 19 \\
\hline Lesiones y envenenamientos (800-999) & 51 & $261(8,5)$ & $4.038(9,7)$ & 7 & 14 & 23 \\
\hline Intervenciones quirúrgicas (00-99) & 46 & $3(0,1)$ & $52(0,1)$ & 13 & 19 & 20 \\
\hline Sin diagnóstico & 53 & $209(6,8)$ & $3.137(7,5)$ & 7 & 12 & 24 \\
\hline Total & 53 & $3.073(100,0)$ & $41.834(100,0)$ & 6 & 12 & 19 \\
\hline
\end{tabular}

*Percentil 25, Mediana, Percentil 75, ${ }^{\dagger}$ Complicaciones del embarazo, parto o puerperio

\section{DISCUSIÓN}

Debido a la falta del diagnóstico en el registro de la IP, este es posiblemente el primer estudio que cuantifica la carga de enfermedad por IP hasta donde sabemos. Por ello, a pesar de que la imputación del diagnóstico no es exacta (de hecho lo razonable sería disponer del diagnóstico de manera sistemática), este estudio proporciona una aproximación de la carga de enfermedad por
IP. Mostrando que los trastornos musculoesqueléticos y mentales constituyen las patologías con mayor carga de la enfermedad por IP. Unos resultados que son coherentes con el estudio internacional sobre carga global de la enfermedad en $2010^{19}$, en el que también se observó que la lumbalgia (el principal trastorno musculo-esquelético) y la depresión mayor (lo mismo para los trastornos mentales) fueron las dos principales causas de años de vida con discapaci- 
dad en países de renta alta ${ }^{20}$ y estuvieron entre las cuatro principales patologías que causan mayor carga, medida mediante disability-adjusted life years (DALYs). Nuestros resultados también concuerdan con los de la cohorte francesa GAZEL ${ }^{21}$ y una cohorte Sueca ${ }^{22}$, encontrándose en ambos estudios un mayor riesgo de desarrollar una IP si la IT previa se debía a diagnósticos psiquiátricos, musculo-esqueléticos o cardiovasculares. Sin embargo, otro posible criterio de imputación como sería el número de episodios de IT previos que algunos estudios encuentran asociados a la $\mathrm{IP}^{18}$, no se utilizó en nuestro estudio por falta de una asociación con la IP. Finalmente, aunque la posible utilización de distintos puntos de corte para establecer si imputamos o no el diagnóstico de la IT a la IP podría incrementar o disminuir el número de IP con diagnóstico probable, el patrón final de los diagnósticos que más frecuentemente ocasionan una IP es bastante estable en las simulaciones realizadas (datos disponibles previa solicitud).

Por otro lado, estudios sobre la carga de enfermedad realizados en España encuentran que las causas con mayor carga medidas por DALYs fueron los trastornos mentales, neoplasias y enfermedades cardiovasculare $^{23}$. Unos resultados que, a diferencia de los nuestros, están basados en el conjunto de la población y resumen tanto la discapacidad como la mortalidad. El estudio de enfermedades crónicas en población activa tiene especial interés ante de los cambios que se están produciendo en el mercado laboral, especialmente en España y en el conjunto de Europa, donde se está experimentando una creciente tasa de desempleo juvenil que retrasa la entrada al mercado laboral de los jóvenes ${ }^{24}$, el incremento de la edad de jubilación ${ }^{2}$ y los cambios en las condiciones laborales con un incremento de la flexibilidad laboral externa (cambios entre empresas) ${ }^{25}$. En este sentido, un reciente estudio que también utiliza la MCVL, encuentra que las personas con una categoría ocupacional manual y no cualificada, al igual que si tiene numerosos contratos temporales, presentan significativamente más APVLP debido a la IP (sin diagnósticos) que las personas con una categoría ocupacional no manual y cualificada, o con una trayectoria más estable ${ }^{26}$.

Como principal limitación de este estudio hemos de señalar los criterios utilizados para imputar el diagnóstico de la IT al de la IP, pues son criterios ad hoc no validados. Sin embargo, consideramos que estos criterios son razonables y posiblemente conservadores. En todo caso, no pretendemos que sean utilizados rutinariamente, para lo que sí habría que validarlos, sino para realizar una primera aproximación a la carga de enfermedad por IP. Asimismo, es una limitación la falta de información sobre algún otro episodio de IT que se haya podido producir después del 2009, o antes, y con diferente diagnóstico y que podría tener relación con una IP iniciada en 2011 o 2012, por ejemplo. Sin embargo, es importante tener en cuenta que el $66 \%$ de las IP con diagnóstico imputado se produjeron en el período 2009-2010 y un menor porcentaje se reconocieron en el 2011y 2012. Es importante recalcar que los $1.665(35,1 \%)$ individuos con IP excluidos del análisis también representan una carga por enfermedad (23.777 APVLP), pero no se les pudo asignar diagnóstico por no cumplir los criterios estipulados y por falta de información sobre la causa de su IP.

Dado el evidente interés que tiene poder estimar los APVLP por IP, parece razonable que la MCVL incluyera el diagnóstico de la IP o al menos estuviera disponible para los investigadores, contribuyendo así a la evaluación de las políticas de salud y de seguridad social.

\section{BIBLIOGRAFÍA}

1. Fries JF. Aging, Natural Death, and the Compression of Morbidity. N Engl J Med. 1980;303:130-5 
2. Sánchez SF. Un estudio en torno a la edad de jubilación. Madrid: Fundación Alternativas. 2011. Disponible en: http://www.falternativas.org/en/estudios-de-progreso/documentos/documentos-de-trabajo/un-estudio-entorno-a-la-edad-de-jubilacion

3. OECD. Sickness, Disability and Work: Breaking the Barriers: A Synthesis of Findings across OECD Countries. París: OECD Publishing; 2010.

4. Castejón J, Benavides FG, Gimeno D, Company A, Fàbrega O, Funes J. Calidad del diagnóstico médico en la certificación de la incapacidad temporal por enfermedad común y accidente no laboral. Aten Primaria. 2006;37:142-7.

5. Ministerio de Empleo y Seguridad Social. Incapacidad Permanente. 2013. Disponible en: http://www.seg-social.es/prdi00/groups/public/documents/binario/47093.p df

6. Ministerio de Empleo y Seguridad Social. Incapacidad temporal. 2013. Disponible en: http://www.segsocial.es/prdi00/groups/public/documents/binario/47197.pdf

7. Benavides FG, Durán X, Miguel Martínez J, Jódar P, Boix P, Amable M. Incidencia de incapacidad permanente en una cohorte de trabajadores afiliados a la Seguridad Social, 2004-2007. Gac Sanit. 2010;24:385-90.

8. Ministerio de Empleo y Seguridad Social. Seguridad Social: Proyecto de presupuestos. Ejercicio 2014. Informe Económico Financiero. Madrid: Ministerio de Empleo y Seguridad Social; 2014. p. 69-175. Disponible en: http://www.seg-social.es/prdi00/groups/public/documents/binario/177768.pdf

9. Duran X, Martínez JM, Benavides FG. Tendencia temporal de la incapacidad laboral permanente en España (1992-2010). Rev Esp Salud Publica. 2012;86:533-542.

10. Overland S, Glozier N, Henderson M, Maeland JG, Hotopf M, Mykletun A. Health status before, during and after disability pension award: the Hordaland Health Study (HUSK). Occup Environ Med. 2008;65:769-73.

11. López MA, Benavides FG, Alonso J, Espallargues M, Durán X, Martínez J. La utilidad del uso de datos administrativos en la investigación de salud pública: la Muestra Continua de Vidas Laborales. Gac Sanit. 2014. Epub 2014 Mar 31

12. Malo MA, Cueto B, García C, Pérez JI. La Medición del Absentismo: Estimaciones desde la perspectiva de las empresas y de las vidas laborales. 2012. Disponible en:http:/www.seg-social.es/prdi00/groups/public/documents/binario/174212.pdf.
13. Lund T, Kivimäki M, Labriola M, Villadsen E, Christensen KB. Using administrative sickness absence data as a marker of future disability pension: the prospective DREAM study of Danish private sector employees. Occup Environ Med. 2008;65:28-31.

14. Virtanen M, Kivimäki M, Vahtera J, Elovainio M, Sund R, Virtanen P, et al. Sickness absence as a risk factor for job termination, unemployment, and disability pension among temporary and permanent employees. Occup Environ Med. 2006;63:212-7.

15. Oyeflaten I, Lie SA, Ihlebæk CM, Eriksen HR. Prognostic Factors for Return to Work, Sickness Benefits, and Transitions Between These States: A 4-year Follow-up After Work-Related Rehabilitation. J Occup Rehabil. 2013.

16. Gjesdal S, Bratberg E. The role of gender in longterm sickness absence and transition to permanent disability benefits. Results from a multiregister based, prospective study in Norway 1990-1995. Eur J Public Health. 2002;12:180-6.

17. Krokstad S, Johnsen R, Westin S. Social determinants of disability pension: a 10-year follow-up of 62 000 people in a Norwegian county population. Int J Epidemiol. 2002;31:1183-91.

18. Wallman T, Wedel H, Palmer E, Rosengren A, Johansson S, Eriksson $\mathrm{H}$, et al. Sick-leave track record and other potential predictors of a disability pension. A population based study of 8,218 men and women followed for 16 years. BMC Public Health. 2009;9:104.

19. Mathers CD, Vos T, Lopez AD, Salomon J, Ezzati M (ed.) National Burden of Disease Studies: A Practical Guide. Edition 2.0. Global Program on Evidence for Health Policy. Geneva: World Health Organization; 2001.

20. Global Burden of Disease Institute for Health Metrics and Evaluation. Disponible en: http://www.healthmetricsandevaluation.org/gbd/visualizations/gbd-arrow-diagram.

21. Alexanderson K, Kivimäki M, Ferrie JE, Westerlund H, Vahtera J, Singh-Manoux A, et al. Diagnosisspecific sick leave as a long-term predictor of disability pension: a 13-year follow-up of the GAZEL cohort study. J Epidemiol Community Health. 2012;66:155-9.

22. Kivimäki M, Ferrie JE, Hagberg J, Head J, Westerlund H, Vahtera J, et al. Diagnosis-specific sick leave as a risk marker for disability pension in a Swedish population. $\mathrm{J}$ Epidemiol Community Health. 2007;61:915-20. 
23. Gènova-Maleras R, Álvarez-Martín E, Morant-Ginestar C, Fernández de Larrea-Baz N, Catalá-López F. Measuring the burden of disease and injury in Spain using disability-adjusted life years: an updated and policy-oriented overview. Public Health. 2012;126:1024-31.

24. Hurley J, Storrie D, Jungblut J. Shifts in the job structure in Europe during the Great Recession.European Foundation for the Improvement of Living and Working Conditions. Luxembourg: Publications Office of the European Union; 2011.p.17-40.

25. Oesch D, Rodriguez Menes J. Upgrading or polarization? Occupational change in Britain, Germany, Spain and Switzerland, 1990-2008. Socio-Economic Rev. 2010;9:503-531.

26. Duran X, Martínez JM, Benavides FG. Occupational factors associated with the potential years of working life lost due to a non-work related permanent disability. Work. 2013;45:305-9. 